\title{
Alteração dimensional linear de resinas para bases de próteses polimerizadas com microondas
}

\section{Linear dimensional change of denture base resins cured by microwave activation}

\author{
Katia Olmedo BRAUN* \\ Renata Cunha Matheus RODRIGUES GARCIA** \\ Célia Marisa RIZZATTI-BARBOSA** \\ Altair Antoninha DEL BEL CURY***
}

\begin{abstract}
BRAUN, K. O.; RODRIGUES GARCIA, R. C. M.; RIZZATTI-BARBOSA, C. M.; DEL BEL CURY, A. A. Alteração dimensional linear de resinas para bases de próteses polimerizadas com microondas. Pesqui Odontol Bras, v. 14, n. 3 , p. $278-282$, jul./set. 2000.
\end{abstract}

\begin{abstract}
A proposta deste estudo foi comparar as alterações dimensionais de 3 resinas submetidas à polimerização com os seguintes ciclos: 1) banho d'água em $73^{\circ} \mathrm{C}$ durante 12 horas - ciclo longo, 2) banho d'água durante 3 horas - ciclo curto, 3) energia de microondas por 3 minutos a potência de $500 \mathrm{~W}$, após armazenamento em água destilada a $37 \pm 2^{\circ} \mathrm{C}$ durante 30 dias. As resinas usadas foram Clássico, Lucitone 550 e Acron MC. As resinas Clássico e Lucitone 550 foram submetidas aos três ciclos, enquanto a resina Acron-MC somente ao ciclo 3. Amostras medindo 65,0 x 10,0 x 3,0 mm e com marcas iguais em três pontos eqüidistantes foram confeccionadas a partir da inclusão de matrizes de aço em gesso-pedra tipo III. Foram confeccionados 21 corpos-de-prova para cada combinação resina/ciclo de polimerização e que foram submetidos a 3 leituras para cada posição (A e B), com o auxílio do microscópio de mensuração (precisão de $0,001 \mathrm{~mm})$, imediatamente após a polimerização e depois do período de imersão em água por 30 dias. Os resultados mostraram que todas as resinas, independendo do ciclo a que tenham sido submetidas e das medidas avaliadas, apresentaram expansão dimensional após terem sido mantidas em água; a composição da resina interferiu na expansão dimensional mais do que o ciclo de polimerização ao qual as resinas foram submetidas; a resina convencional, quando polimerizada com energia de microondas, apresentou alteração dimensional semelhante à resina desenvolvida para a polimerização em microondas.
\end{abstract}

UNITERMOS: Resinas acrílicas; Polímeros.

\section{INTRODUÇÃO}

Mesmo considerando-se os avanços da Odontologia Preventiva e Restauradora, inclusive vislumbrando a possibilidade do emprego de implantes ósseo-integrados, faz-se necessária, ainda hoje, a utilização de próteses parciais removiveis e totais para substituir dentes naturais ausentes, reabilitando estética e funcionalmente um grande número de pacientes.

Esses trabalhos protéticos tradicionalmente têm sido confeccionados com resina acrílica termopolimerizável que pode sofrer contração de polimerização durante o processamento das bases das próteses ${ }^{1}$. Essa contração de polimerização produz estresse interno que é liberado durante os procedi- mentos de demuflagem podendo resultar em alteração dimensional e distorção dessas bases ${ }^{2}$. Com vistas à resolução desses problemas, vários materiais e técnicas de processamento têm sido introduzidos na Odontologia com a finalidade de obtenção de próteses com melhor adaptação e resistência ${ }^{7}$. Assim, pesquisas foram conduzidas objetivando a utilização de energia de microondas como fonte alternativa no processamento das resinas acrílicas ${ }^{4,5,6}$. Também foi demonstrado por REITZ et al. ${ }^{8}$ (1985); DE CLERCK ${ }^{3}$ (1987) e TAKAMATA; SETCOS ${ }^{11}$ (1989) significante melhoria na manipulação desse material, tanto no uso clínico quanto na precisão dimensional obtida por essa técnica. As microondas geradas pelo magnétron do

\footnotetext{
* Professora Assistente do Departamento de Odontologia Restauradora da Universidade Federal de Santa Maria.

** Professoras Assistentes Doutoras do Departamento de Prótese e Periodontia; *** Professora Livre-Docente da Área de Prótese Parcial Removível - Faculdade de Odontologia de Piracicaba da UNICAMP.
} 
BRAUN, K. O.; RODRIGUES GARCIA, R. C. M.; RIZZATTI-BARBOSA, C. M.; DEL BEL CURY, A. A. Alteração dimensional linear de resinas para bases de próteses polimerizadas com microondas. Pesqui Odontol Bras, v. 14, n. 3, p. 278-282, jul./set. 2000.

forno de microondas promovem o aquecimento dielétrico no qual a energia é imediatamente absorvida pela resina de maneira uniforme, eliminando-se o tempo necessário para transferir o calor da água quente para a mufla, desta para o gesso de revestimento e por fim para a resina, fazendo com que esse processo seja rápido, fácil e limpo ${ }^{4,12}$. Entretanto, existem opiniões controvertidas se este tipo de processamento proporcionaria bases de próteses com menor alteração dimensional ${ }^{3,10,11}$, quando comparado ao método convencional da água aquecida.

Considerando que a adaptação das bases de próteses está relacionada com as alterações que as resinas acrílicas podem sofrer durante o ciclo de polimerização ${ }^{2}$ e que essa contração de polimerização pode ser compensada com a sorção de água ${ }^{6}$, foi objeto deste estudo verificar e comparar as alterações dimensionais lineares de três resinas para bases de próteses, quando submetidas a três ciclos de polimerização: A- longo; B- curto e, C- energia de microondas, quando imersas em água destilada por um período de trinta dias.

\section{MATERIAL E MÉTODO}

Foram utilizadas três marcas comerciais de resinas acrilicas: 1) Acron MC (G.C. Dental Ind. Corp.), resina para polimerização em microondas; 2) Clássico (Clássico Artigos Odontológicos Ltda.) e 3) Lucitone 550 (Dentsply Ind. e Com. Ltda.) - resinas termopolimerizáveis convencionais.

\section{Confecção das amostras}

Os corpos-de-prova foram confeccionados a partir da inclusão de matrizes de aço com as dimensões de 65,0 x 10,0 x 3,0 mm em gesso-pedra tipo III. Estas matrizes possuíam marcas iguais em três pontos eqüidistantes (Figura 1) que permitiriam as mensurações para a determinação da presença ou não das alterações dimensionais. Após a presa do gesso, as matrizes foram retiradas, per-

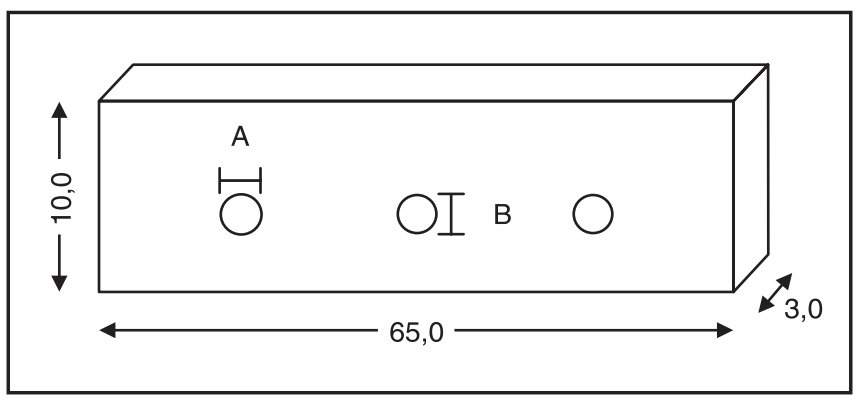

FIGURA 1 - Esquema representativo da amostra e medidas realizadas nas posições A e B. manecendo o molde para a confecção das amostras.

A seguir todas as resinas foram preparadas conforme as instruções dos fabricantes e na fase plástica inseridas nos moldes. As muflas foram fechadas e comprimidas lentamente até que ambas as partes se encontrassem em prensas de banca$\mathrm{da}$, onde permaneceram por trinta minutos em temperatura ambiente. Decorrido este período as resinas Clássico e Lucitone 550 foram submetidas aos 3 ciclos de polimerização e a resina Acron MC somente ao ciclo 3:

- Ciclo 1: 12 horas em polimerizadora automática à temperatura de $73 \pm 2{ }^{\circ} \mathrm{C}$;

- Ciclo 2: 90 minutos à temperatura de $65 \pm 3^{\circ} \mathrm{Ce}$ 90 minutos à aproximadamente $100^{\circ} \mathrm{C}$;

- Ciclo 3: 3 minutos a $500 \mathrm{~W}$ em forno de microondas.

A polimerizadora utilizada para o ciclo longo (ciclo 1) foi a de marca Righeto e a usada para o ciclo curto (ciclo 2) foi a de marca Thermotron. Para o ciclo 3 as muflas contendo as resinas foram levadas individualmente ao forno de microondas com prato giratório modelo BFP $61 \mathrm{~F}$ com potência de $1.240 \mathrm{~W}$ (Brastemp Ltda.) por 3 minutos em potência média que corresponde aproximadamente a $500 \mathrm{~W}$. Todas as muflas, depois de completado o ciclo de polimerização, permaneceram sobre a bancada de trabalho até completo resfriamento, e posteriormente as amostras foram submetidas ao acabamento com lixas d'água com granulação decrescente 400, 500 e 600 até atingir as dimensões predeterminadas de $65,0 \times 10,0 \times 3,0 \mathrm{~mm}$.

\section{Avaliação da alteração dimensional}

Foram confeccionados 21 corpos-de-prova para cada combinação ciclo/resina, codificados e submetidos a 3 leituras para cada posição (A e B) (Figura 1) por um mesmo operador em microscópio de mensuração com precisão de 0,001 mm (Ernest Leitz - Wetzlar, Germany), sendo que para cada 3 leituras foi feita a média para cada medida. As leituras foram realizadas antes e após a imersão em água por 30 dias. Os resultados obtidos foram submetidos a análise estatística. Foi feita uma análise de variância e teste de Tukey com 95\% de confiança $(\alpha=0,05)$, comparando-se as três resinas em todos os ciclos quanto à alteração dimensional. Para a comparação das médias obtidas antes e após a imersão em água, foi usado o teste $t$ pareado. 
BRAUN, K. O.; RODRIGUES GARCIA, R. C. M.; RIZZATTI-BARBOSA, C. M.; DEL BEL CURY, A. A. Alteração dimensional linear de resinas para bases de próteses polimerizadas com microondas. Pesqui Odontol Bras, v. 14, n. 3, p. 278-282, jul./set. 2000.

TABELA 1 - Médias e Desvios-Padrão da alteração dimensional linear das resinas imediatamente após a demuflagem e 30 dias após imersão em água - Posições A e B.

\begin{tabular}{|c|c|c|c|c|c|c|c|c|c|}
\hline \multirow[b]{2}{*}{ Resinas } & \multirow[b]{2}{*}{ Ciclo } & \multicolumn{4}{|c|}{ Posição A } & \multicolumn{4}{|c|}{ Posição B } \\
\hline & & Imediato & $\mathrm{DP}$ & 30 dias & $\mathrm{DP}$ & Imediato & $\mathrm{DP}$ & 30 dias & DP \\
\hline \multirow{3}{*}{ Clássico } & 1 & 3,378 & 0,042 & $3,416^{*}$ & 0,075 & 3,297 & 0,060 & $3,393^{*}$ & 0,040 \\
\hline & 2 & 3,371 & 0,038 & $3,392 *$ & 0,040 & 3,298 & 0,056 & $3,371^{*}$ & 0,059 \\
\hline & 3 & 3,372 & 0,411 & $3,420 *$ & 0,085 & 3,372 & 0,081 & $3,451 *$ & 0,086 \\
\hline \multirow{3}{*}{ Lucitone 550} & 1 & 3,358 & 0,069 & $3,448 *$ & 0,079 & 3,355 & 0,061 & $3,435^{*}$ & 0,074 \\
\hline & 2 & 3,283 & 0,037 & $3,407 *$ & 0,074 & 3,257 & 0,033 & $3,425^{*}$ & 0,084 \\
\hline & 3 & 3,323 & 0,028 & $3,442 *$ & 0,078 & 3,295 & 0,040 & $3,433^{*}$ & 0,071 \\
\hline Acron $\mathrm{MC}$ & 3 & 3,402 & 0,041 & $3,428^{*}$ & 0,087 & 3,366 & 0,038 & $3,435^{*}$ & 0,079 \\
\hline
\end{tabular}

* Diferença significante $(\mathrm{p}<0,05)$ pelo teste $t$ pareado após armazenagem em água.

TABELA 2 - Comparação das Médias da alteração dimensional linear das resinas Clássico e Lucitone 550 em todos os ciclos de polimerização - Posições A e B.

\begin{tabular}{l|c|c|c|c|c|c|c|c}
\hline \hline & \multicolumn{4}{|c|}{ Posição A } & \multicolumn{4}{c}{ Posição B } \\
\hline \multicolumn{1}{c|}{ Resinas } & Ciclo 1 & Ciclo 2 & Ciclo 3 & Média & Ciclo 1 & Ciclo 2 & Ciclo 3 & Média \\
\hline Clássico & 0,038 & 0,021 & 0,048 & $0,035 \mathrm{~B}$ & 0,096 & 0,093 & 0,080 & $0,089 \mathrm{~A}$ \\
\hline Lucitone 550 & 0,090 & 0,124 & 0,085 & $0,099 \mathrm{~A}$ & 0,080 & 0,168 & 0,110 & $0,119 \mathrm{~A}$ \\
\hline Média & $0,064 \mathrm{a}$ & $0,072 \mathrm{a}$ & $0,066 \mathrm{a}$ & & $0,088 \mathrm{a}$ & $0,130 \mathrm{a}$ & $0,095 \mathrm{a}$ & \\
\hline \hline
\end{tabular}

Médias seguidas de letras distintas (maiúscula na vertical e minúscula na horizontal) diferem entre si pelo teste de Tukey $(\mathrm{p}<0,05)$.

\section{RESULTADOS}

Pode-se observar na Tabela 1 que houve aumento significativo $(\mathrm{p}<0,05)$ para as posições A e B para todas as resinas e todos os ciclos de polimerização após 30 dias de imersão das amostras em água destilada.

A interação entre as resinas acrílicas utilizadas e os ciclos de polimerização está apresentada na Tabela 2. Nota-se que, com relação aos ciclos de polimerização, os resultados não diferiram estatisticamente para as posições A e B. No entanto, para o fator resina, a Lucitone 550 diferiu significantemente da resina Clássico $(\mathrm{p}<0,05)$, apresentando maior alteração dimensional na posição A.

Com relação à interação resina acrílica e ciclo de polimerização para a posição B (Tabela 2), independente do ciclo utilizado não houve diferença significativa ( $p>0,05)$ quanto à alteração dimensional linear, para as resinas Clássico e Lucitone 550.

Para o ciclo de polimerização 3, a resina Lucitone 550 foi a que apresentou maior alteração dimensional na posição A (Tabela 3), entretanto
TABELA 3 - Comparação da alteração dimensional linear de todas as resinas no ciclo 3 - Posições A e B.

\begin{tabular}{l|c|c}
\hline \hline \multicolumn{1}{c|}{ Resinas } & Posição A & Posição B \\
\hline Clássico & $0,048 \mathrm{AB}$ & $0,080 \mathrm{~A}$ \\
\hline Lucitone 550 & $0,085 \mathrm{~A}$ & $0,110 \mathrm{~A}$ \\
\hline Acron MC & $0,026 \mathrm{~B}$ & $0,068 \mathrm{~A}$ \\
\hline \hline
\end{tabular}

Médias seguidas de letras distintas nas colunas diferem entre si pelo teste de Tukey $(\mathrm{p}<0,05)$.

diferiu significativamente somente da resina Acron MC ( $\mathrm{p}<0,05)$. Não foi observada diferença estatisticamente significante entre as resinas Lucitone 550 e Clássico, e entre as resinas Acron MC e Clássico. Para a posição B (Tabela 3) os resultados não diferiram estatisticamente entre as três resinas estudadas $(\mathrm{p}>0,05)$.

\section{DISCUSSÃO}

Os resultados da Tabela 1 mostram que todas as resinas, independente do ciclo a que tenham sido submetidas e das posições avaliadas (A ou B), apresentaram expansão dimensional após terem 
BRAUN, K. O.; RODRIGUES GARCIA, R. C. M.; RIZZATTI-BARBOSA, C. M.; DEL BEL CURY, A. A. Alteração dimensional linear de resinas para bases de próteses polimerizadas com microondas. Pesqui Odontol Bras, v. 14, n. 3, p. 278-282, jul./set. 2000.

sido mantidas em água durante 30 dias, estando de acordo com os resultados de RIZZATTIBARBOSA et al..$^{9}$ (1995) o que poderia ser explicado devido a sorção de água pelas resinas.

Quando se compara a resina Clássico com a Lucitone 550 nos três ciclos utilizados no estudo, pode ser verificado na Tabela 2 que não houve diferença estatística significativa entre os ciclos utilizados para a posição A. Entretanto, as duas resinas mostraram-se diferentes entre si para os três ciclos, com menor alteração para a resina Clássico. Esse resultado pode ser explicado pela diferença de composição entre estas resinas, pois a resina Lucitone 550 apresenta agentes de ligação cruzada no monômero que alteram a sorção de água ${ }^{2}$.

Quando se trata da posição B, observa-se na Tabela 2 que as duas resinas continuam não apresentando diferença estatística significativa entre os ciclos utilizados, porém apresentam o mesmo comportamento, ou seja, a resina Clássico apresentou as menores alterações. Isso talvez possa ser explicado pela diferença de volume de resina que existe entre as demarcações, pois há uma maior quantidade de resina no sentido da posição A, quando comparada com a quantidade de resina existente no sentido da posição B. Assim, ocorre um menor volume de resina para a posição B facilitando, dessa forma, a difusão de água e proporcionando um maior aumento de volume nesse período de armazenagem, pois a difusão da água ocorre entre as macromoléculas forçando ligeiramente o seu afastamento ${ }^{2}$.

Na Tabela 3, estão comparadas as alterações dimensionais lineares das resinas Clássico, Lucito- ne 550 e Acron MC tanto para a posição A como para a posição $\mathrm{B}$ com relação ao ciclo 3 , e pode-se verificar que a resina Acron MC obteve a menor alteração dimensional seguida da resina Clássico e por fim da resina Lucitone 550. Para a posição A, a resina Acron MC apresentou diferença estatística significativa quando comparada com a resina $\mathrm{Lu}-$ citone 550, porém não apresentou diferença estatística significativa com a resina Clássico, estando de acordo com os resultados de SANDERS et al. ${ }^{10}$ (1991) que verificaram que a resina desenvolvida para a polimerização com energia de microondas mostrou adaptação tão boa quanto a da resina convencional quando polimerizada em banho de água aquecida e mesmo quando da polimerização dessa resina convencional pela energia de microondas.

\section{CONCLUSÕES}

Diante dos resultados obtidos neste estudo, podemos concluir que:

1. todas resinas acrílicas apresentaram alteração dimensional quando submetidas à imersão em água, independente do ciclo de polimerização a que tenham sido submetidas;

2. a composição e a quantidade de resina interferiram na alteração dimensional quando estas foram armazenadas em água;

3. a resina convencional, quando submetida ao ciclo de polimerização em microondas, apresentou alteração dimensional semelhante à resina desenvolvida para a polimerização em microondas.

BRAUN, K. O.; RODRIGUES GARCIA, R. C. M.; RIZZATTI-BARBOSA, C. M.; DEL BEL CURY, A. A. Linear dimensional change of denture base resins cured by microwave activation. Pesqui Odontol Bras, v. 14, n. 3, p. 278-282, jul./set. 2000.

This study examined the influence of three polymerization cycles (1: heat cure - long cycle; 2 : heat cure - short cycle; and 3: microwave activation) on the linear dimensions of three denture base resins, immediately after deflasking, and 30 days after storage in distilled water at $37 \pm 2^{\circ} \mathrm{C}$. The acrylic resins used were: Clássico, Lucitone 550 and Acron $\mathrm{MC}$. The first two resins were submitted to all three polymerization cycles, and the Acron MC resin was cured by microwave activation only. The samples had three marks, and dimensions of $65 \mathrm{~mm}$ in length, $10 \mathrm{~mm}$ in width and $3 \mathrm{~mm}$ in thickness. Twenty-one test specimens were fabricated for each combination of resin and cure cycle, and they were submitted to three linear dimensional evaluations for two positions (A and B). The changes were evaluated using a microscope. The results indicated that all acrylic resins, regardless of the cure cycle, showed increased linear dimension after 30 days of storage in water. The composition of the acrylic resin affected the results more than the cure cycles, and the conventional acrylic resin (Lucitone 550 and Clássico) cured by microwave activation presented similar results when compared with the resin specific for microwave activation.

UNITERMS: Acrylic resins; Polymers. 
BRAUN, K. O.; RODRIGUES GARCIA, R. C. M.; RIZZATTI-BARBOSA, C. M.; DEL BEL CURY, A. A. Alteração dimensional linear de resinas para bases de próteses polimerizadas com microondas. Pesqui Odontol Bras, v. 14, n. 3, p. 278-282, jul./set. 2000.

\section{REFERÊNCIAS BIBLIOGRÁFICAS}

1. ANUSAVICE, K. J. Denture base resins, technical considerations and processing techniques. In: PHILLIPS' Science of dental materials. 10. ed. Philadelphia : WB Saunders Company, 1996. Cap. 11, p. 237-272.

2. CRAIG, R. G. Prosthetic applications of polymers. In:

Restorative Dental Materials. 10. ed. St Louis : Mosby-Year book, 1997. Cap. 19, p. 500-559.

3. DE CLERCK, J. P. Microwave polymerization of acrylic resins used in dental prostheses. J Prosthet Dent, v. 57, n. 5, p. 650-658, May 1987.

4. DEL BEL CURY, A. A.; RODRIGUES Jr., A. L.; PANZERI, H. Resinas acrílicas dentais polimerizadas por energia de microondas, método convencional de banho de água e quimicamente ativada: propriedades físicas. Rev Odontol Univ São Paulo, v. 8, n. 4, p. 242-249, out./dez. 1995.

5. KIMURA, H.; TERAOKA, F.; SAITO, T. Applications of microwave for dental technique (part I): Dough forming and curing of acrylic resins. J Osaka Univ Dent. v. 23, p. 43-49, Dec. 1983.

6. KIMURA, H.; TERAOKA, F.; SAITO, T. Applications of microwave for dental technique (part II): Adaptability of cured acrylic resins. J Osaka Univ Dent, v. 24, p. 19-21, Dec. 1984.

7. LEVIN, B.; SANDERS, J. L.; REITZ, P. V. The use of microwave energy for processing acrylic resins. J Prosthet Dent, v. 61, n. 3, p. 381-383, Mar. 1989.

8. REITZ, P. V.; SANDERS, J. L.; LEVIN, B. The curing of denture acrylic resin by microwave energy. Physical properties. Quintessence Int, v. 16, n. 8, p. 547-551, Aug. 1995.

9. RIZZATTI-BARBOSA, C. M.; DEL BEL CURY, A. A.; PANZERI, H. Influência da sorção de água e do processo de polimerização por energia de microondas na adaptabilidade de próteses totais. Rev Odontol Univ São Paulo, v. 9, n. 3, p. 197-206, jul./set. 1995.

10. SANDERS, J. L.; LEVIN, B.; REITZ, P. V. Comparison of the adaptation of acrylic resins cured by microwave energy and conventional water bath. Quintessence Int, v. 22, n. 3, p. 181-186, July 1991.

11. TAKAMATA, T.; SETCOS, J. C. Resin dentures bases: Review of accuracy and methods of polymerization. Int $\mathbf{J}$ Prosth, v. 2, n. 6, p. 555-562, Nov./Dec. 1989.

12. WALlACE, P. W.; GRASER, G. N.; MYERS, M. L. et al. Dimensional accuracy of denture resin cured by microwave energy. J Prosthet Dent, v. 66, n. 3, p. 403-408, Sept. 1991.
Recebido para publicação em 16/10/99 Enviado para reformulação em 20/03/00 Aceito para publicação em 02/06/00 